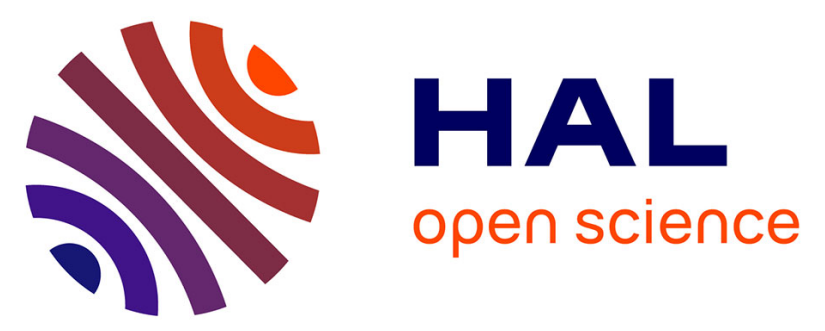

\title{
Token-Based Lightweight Authentication to Secure IoT Networks
}

\author{
Maissa Dammak, Omar Rafik Merad Boudia, Mohamed-Ayoub Messous, \\ Sidi-Mohammed Senouci, Christophe Gransart
}

\section{To cite this version:}

Maissa Dammak, Omar Rafik Merad Boudia, Mohamed-Ayoub Messous, Sidi-Mohammed Senouci, Christophe Gransart. Token-Based Lightweight Authentication to Secure IoT Networks. CCNC 2019, IEEE Consumer Communications \& Networking Conference, Jan 2019, Las vegas, United States. 10.1109/CCNC.2019.8651825 . hal-02002403

\section{HAL Id: hal-02002403 \\ https://hal.science/hal-02002403}

Submitted on 14 Feb 2022

HAL is a multi-disciplinary open access archive for the deposit and dissemination of scientific research documents, whether they are published or not. The documents may come from teaching and research institutions in France or abroad, or from public or private research centers.
L'archive ouverte pluridisciplinaire HAL, est destinée au dépôt et à la diffusion de documents scientifiques de niveau recherche, publiés ou non, émanant des établissements d'enseignement et de recherche français ou étrangers, des laboratoires publics ou privés.

\section{다)(1) $(5$}

Distributed under a Creative Commons Attribution - NonCommerciall 4.0 International 


\title{
Token-Based Lightweight Authentication to Secure IoT Networks
}

\author{
Maissa Dammak ${ }^{1}$, Omar Rafik Merad Boudia ${ }^{2}$, Mohamed Ayoub Messous ${ }^{1}$, Sidi Mohammed Senouci ${ }^{1}$, Christophe Gransart $^{3}$ \\ ${ }^{1}$ DRIVE EA1859, Univ. Bourgogne Franche Comté, France \\ ${ }^{2}$ STIC Laboratory, University of Tlemcen, Algeria \\ ${ }^{3}$ IFSTTAR, COSYS/LEOST Villeneuve d'Ascq, France \\ \{maissa.dammak ; ayoub.messous ; sidi-mohammed.senouci\}@u-bourgogne.fr; om_meradboudia@mail.univ-tlemcen.dz; christophe.gransart@ifsttar.fr
}

\begin{abstract}
The rapid growth of Internet of Things (IoT) technology offers huge opportunities and also brings many new challenges related to the authentication in IoT devices. Using passwords or pre-defined keys have drawbacks that limit their use for different IoT applications like smart hotel and smart office. In fact, they didn't provide temporary access to data in such reservation systems. Thus, authenticating users basing on password mechanism is not feasible. In this paper, we propose a new Token-Based Lightweight User Authentication (TBLUA) for IoT devices, which is based on token technique in order to enhance the robustness of authentication. Security analysis shows the security strength of the proposed scheme such as token security, Perfect Forward Secrecy (PFS), etc. In addition, the presented performance analysis shows that it is a strong competitor among existing ones for user authentication in IoT environments.
\end{abstract}

Keywords-Lightweight Authentication, Token, Security, Perfect Forward Secrecy, De-Synchronization Attack.

\section{INTRODUCTION}

The proliferation of the internet of thing (IoT) have expended into many aspects of our life style and social interactions, the next frontier is digital industry environment (e.g. smart city, smart hotel, smart office) [1]. Although, the future of smart industry environment is promising, many technical challenges must be addressed to achieve convenience and security [2]. Specifically, user lightweight authentication for reservation system has been a critical issue due to the communication between the user and smart devices which is limited in time. The reason is that the users may want to reserve a list of smart devices to establish communications for a period of time. For this purpose, it is important to authenticate the legitimacy of a user for a predefined time interval. In this context, tokens have been introduced as an efficient solution to create a strong binding between the users that requested the reservation and the smart device. At the same time, token-based authentication reduces the risk of stolen authentication factors as tokens are protected against misuse, and it does not require much more user effort than password-based mechanism [3].

The user-to-device authentication is fundamental, however, most of IoT devices are resource-constrained devices and they need to transmit sensed data periodically. Hence, it is necessary for smart things to adopt a lightweight authentication protocol to reduce their energy consumption when a device aims to authenticate and transmit data to its targeted peer. Likewise, IoT devices communicate over insecure communication channels and an illegal user (attacker) can break the security and also gain access over the smart device [4][5]. Furthermore, by compromising one secret key, an attacker may deduce any previous session key which represents a serious threat. Thus, Perfect Forward
Secrecy is a basic security property for session key-based authentication [6].

To the best of our knowledge, most of the authentication schemes have several security limitations especially PFS, which is the basic and important security property for authentication in IoT environments. Besides, all most previously proposed schemes are based on two or threefactor authentication [5][8][9][12] which limit their use in reservation system.

In this paper, we propose a lightweight authentication protocol based on token technique to reach the design goals. This protocol:

- Generates an additional security layer of authentication by adopting the token technique which offers access to a specific resource for a predefined period of time.

- Reduces the computation overhead and save energy for authenticating devices during the authentication session, by using only lightweight computation operations such as XOR and hash function.

- Is designed to withstand the most popular security attacks and ensures the known security property especially PFS.

- Proves, by simulating its performance with existing schemes, that it is more efficient and lightweight solution.

The rest of the paper is structured as follows. The network model and the threat model are presented in section II. The proposed scheme TBLUA for user authentication in IoT environments is presented in Section III. Informal security analysis and the performance comparison with the existing relevant schemes are given in Section IV. Finally, Section VII concludes this paper.

\section{SYSTEM MODEL}

In this model, we have followed two models which are discussed below:

\section{A. Network and threat models}

In this section we present the network model which is depicted in Fig.1. Our model consists of the end user $U_{i}$ who needs to register herself/himself at the trusted Reservation Server (RS) in order to communicate with Smart Devices $\mathrm{SD}_{\mathrm{j}}$. RS is responsible for generating reservation tokens for $U_{i}$ and distribute them for the Registration Authority (RA). The latter is responsible for registering all smart devices and gateway (GW) securely. Moreover, we assume that all the heterogeneous devices are synchronized with their clocks and agree a maximum transmission delay $(\Delta T)$ to protect our scheme against replay attacks [10]. We have used the DolevYao threat model [9], in which two communicating parties $\left(U_{i}, S D_{j}\right)$ interact over insecure channel and they are not considered as trustworthy. An adversary, let's call it $A$, can 
eavesdrop the exchanged messages, and thus modify or delete the messages during transmission. Furthermore, $S D_{j}$ are not tamper-resistant and thus, they can be physically compromised by $A$. Also, the user's smart phone $S P$ can be lost/stolen by $A$. Therefore, $A$ can extract sensitive information stored in those nodes using the well-known power analysis attacks [13]. Nevertheless, we assume that the $\mathrm{GW}$ in the proposed scheme is a trusted node and is not compromised under any circumstances; otherwise, the whole network is compromised [5]. Furthermore, RA and RS are also fully trusted and cannot be compromised by an adversary.

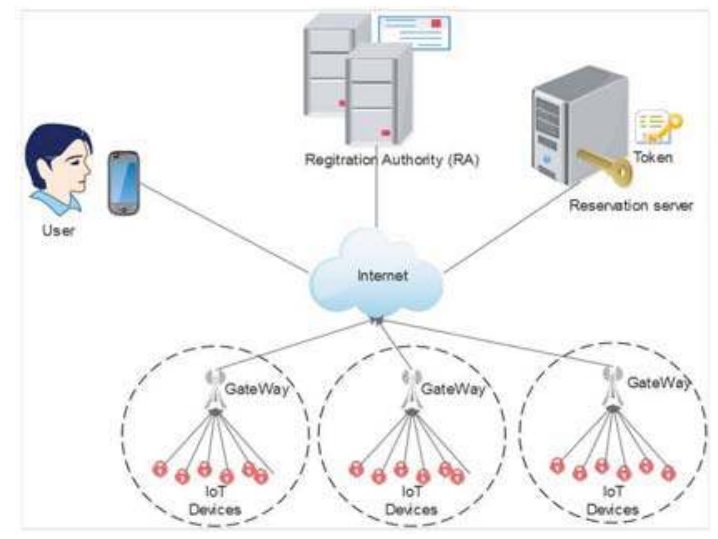

Fig.1. Proposed NeTWORK MODEL

\section{TBLUA SCHEME DESCRIPTION}

In this section, we describe the proposed authentication and key negotiation protocol to make secure data transmission after a successful reservation. The proposed authentication protocol includes the following phases: (i) Offline smart device and GW registration, (ii) User reservation, (iii) Token distribution between GW and smart devices and (iv) Login and Authentication. All these phases are detailed in the following subsections. Notations are presented in Table I.

\begin{tabular}{|c|l|}
\multicolumn{2}{c}{ TABLE I. $\quad$ SYMBOLS AND THEIR DESCRIPTIONS } \\
\hline Symbols & \multicolumn{1}{c|}{ Descriptions } \\
\hline$R S$ & Reservation Server \\
$U_{i}$ & Registration Authority \\
$G W$ & User \\
$S D_{j}$ & Sateway node \\
$P W_{i}$ & Password of $U_{i}$ \\
$I D_{i}$ & Identity of $U_{i}$ \\
$S P$ & User's Smart Phone \\
$I D_{S D j}$ & Identity of $S D_{j}$ \\
$K$ & Secret key of GW \\
$K_{U G}$ & Shared key between $U_{i}$ and GW \\
$K_{S G}$ & Shared key between $S D_{j}$ and GW \\
$T I D_{i}$ & Temporary identity generated by GW for $U_{i}$ \\
$R_{1}$ & Random nonce created by $U_{i}$ \\
$R_{2}$ & Random nonce created by GW \\
$R_{3}$ & Random nonce created by $S D_{j}$ \\
$E K(\cdot) / D K(\cdot)$ & Symmetric encrypt/decrypt using key $K$ \\
$N S_{j}$ & Sequence number \\
$T_{i}, t_{i}$ & Current timestamp \\
$\Delta T$ & Maximum transmission delay \\
$h(\cdot)$ & Cryptographic one-way hash function \\
$\|, \oplus$ & Concatenation operation, Bitwise XOR operation \\
\hline
\end{tabular}

\section{A. Offline smart device and $G W$ registration phase}

The offline sensing node registration phase is executed by the RA. The RA selects a unique identity $I D_{S D j}$ for each deployed smart device $S D_{j}$ and also generates a unique random 160-bits secret shared key, $K_{S G}$, between the GW and
$\mathrm{SD}_{i}$, where $1 \leq j \leq n$ ( $n$ is the number of smart devices) and the initial sequence numbers $N S_{j}=N S_{j 0}=0$. The RA stores $\left\{I D_{S D j}, N S_{j}, K_{S G}\right\}$ into the smart device $S D_{j}$ memory, and $\left\{I D_{S D j}, N S_{j 0}, K_{S G}\right\}$ into the GW memory.

The RA further randomly generates a unique GW's identity $I D_{G W}$ and a unique random 1024-bit secret key $K$. RA defines a group of $S D_{j}$ which is identified by $G_{i}=\left\{S D_{j} ; 1<\mathrm{j}<\right.$ $\mathrm{N}, \mathrm{N}$ is the number of $S D_{j}$ in $\left.G_{i}\right\}$ and computes $\mathrm{S}_{\mathrm{j}}=\mathrm{h}\left(I D_{S D_{j}} \|\right.$ $\left.G_{i} \| K\right)$ for each $S D_{j}$ and updates the $S D_{j}$ node information table entry with $<I D_{S D j}, S_{j}, N S_{j 0}, K_{S G}, G_{i}>$ in the GW memory.

\section{B. User reservation phase}

To access the services from a particular smart device $S D_{j}$, a user $U_{i}$ first needs to register with the RA securely. The following steps in Table II are required for this registration:

TABLE II. SUMMARY OF USER RESERVATION PHASE

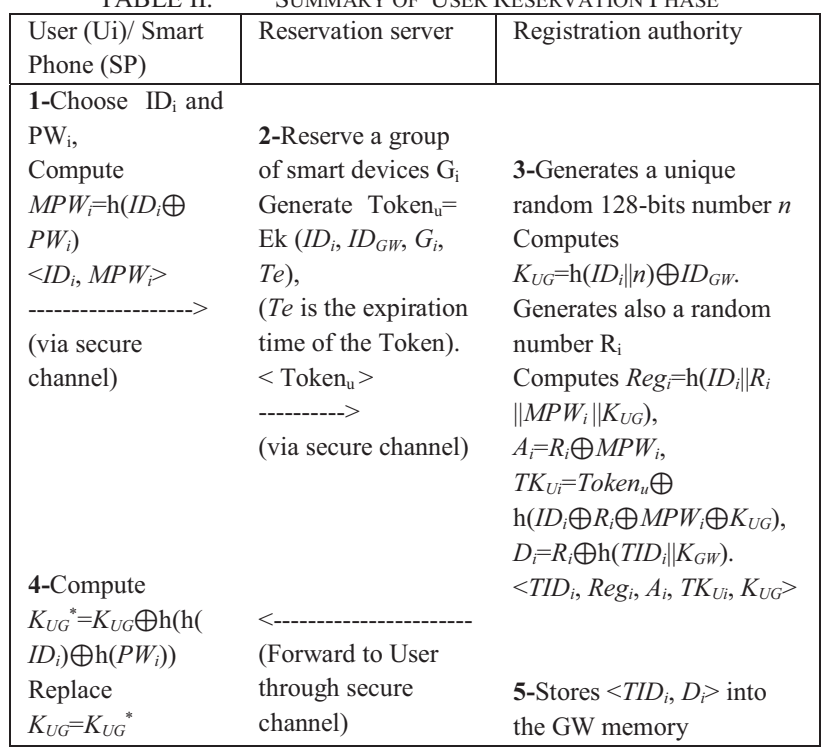

C. Token distribution between $G W$ and smart device phase

In this phase, the GW distributes periodically the token of a user Ui to a group of smart devices after a successful reservation phase. It is detailed on 4 steps as follows:

\begin{tabular}{|c|c|}
\hline Gateway node (GW) & Smart Device $\left(\mathrm{SD}_{\mathrm{j}}\right)$ \\
\hline 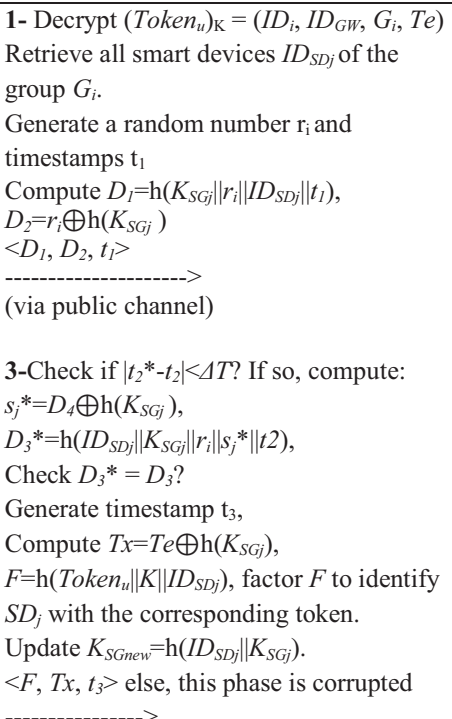 & $\begin{array}{l}\text { 2-Checks if }\left|t_{1}{ }^{*}-t_{l}\right|<\Delta T \text { ? } \\
\text { If so, computes } \\
r_{i}^{*}=D_{2} \oplus \mathrm{h}\left(K_{S G j}\right), \\
D_{l}^{*}=\mathrm{h}\left(K_{S G j}|| r_{i}{ }^{*}|| I D_{S D j}|| \mathrm{t}_{1}\right), \\
\text { Check if } D_{l}^{*}=D_{l} \text { ? If so, } \\
\text { Generates a random } \\
\text { number } \mathrm{s}_{\mathrm{j}} \text { and } \\
\text { timestamp t } \\
\text { Computes } \\
D_{3}=\mathrm{h}\left(I D_{S D_{j}}|| K_{S G}\left\|r_{i}{ }^{*}\right\| s_{j}|| t_{2}\right) \text {, } \\
D_{4}=S_{j} \oplus \mathrm{h}\left(K_{S G}\right), \\
<D_{3}, D_{4}, t_{2}>, \text { Otherwise, } \\
\text { this phase is corrupted } \\
<---------- \\
\text { (via public channel) } \\
\text { 4-Update } \\
K_{S G n e w}=\mathrm{h}\left(I D_{S D j} \| K_{S G j}\right), \\
\text { Store } F \text { and } T x \text { in its } \\
\text { memory }\end{array}$ \\
\hline
\end{tabular}


TABLE IV. SUmmary of Login ANd Authentication Phase

\begin{tabular}{|c|c|c|}
\hline User (Ui)/ Smart Phone (SP) & Gateway node (GWN) & Smart device (SDi) \\
\hline 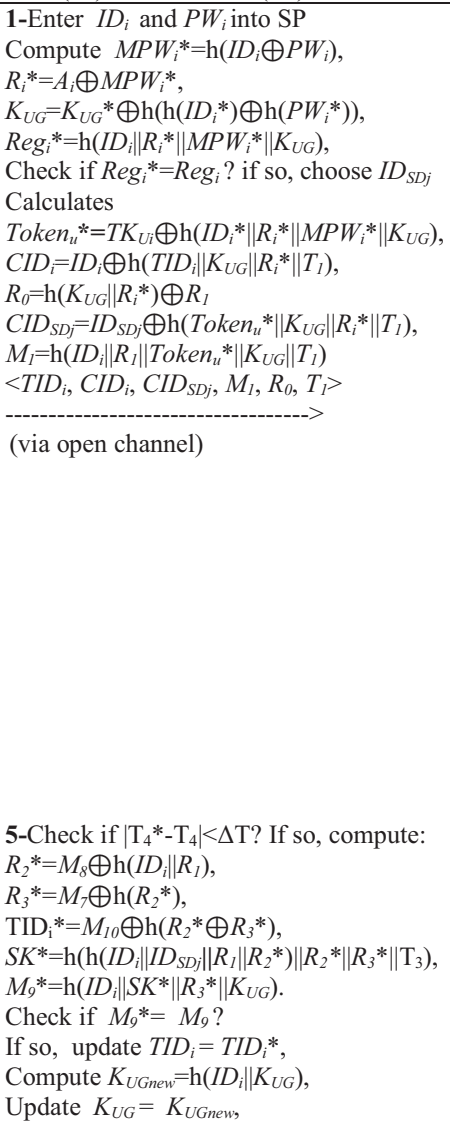 & 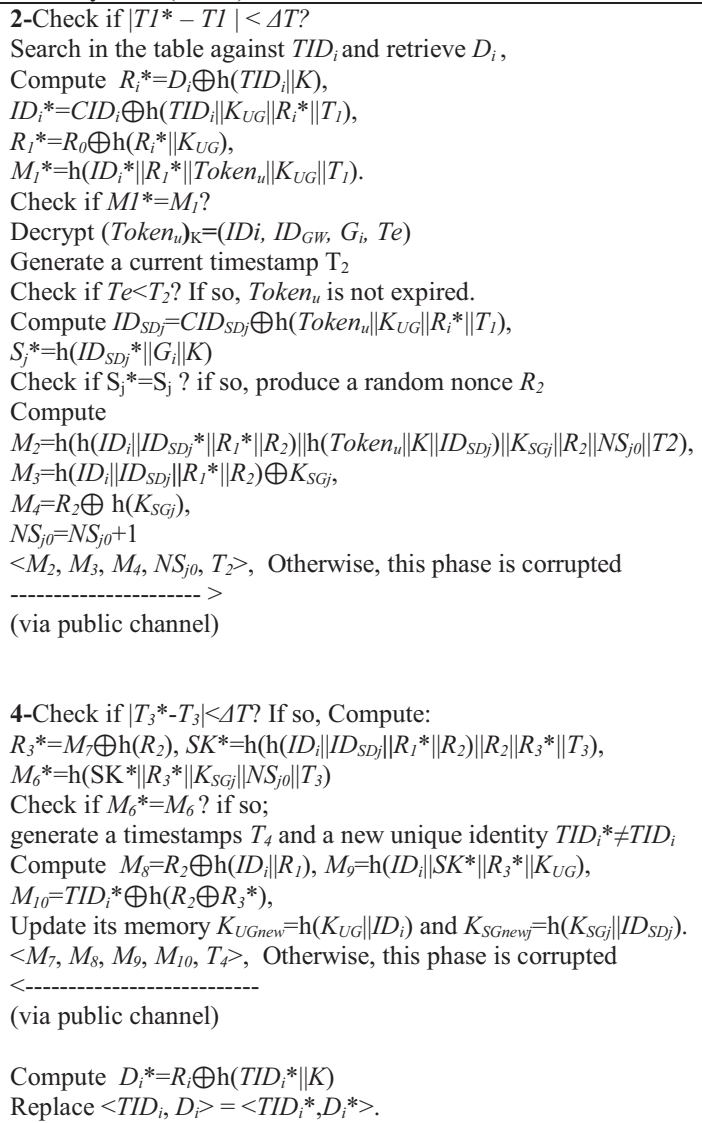 & 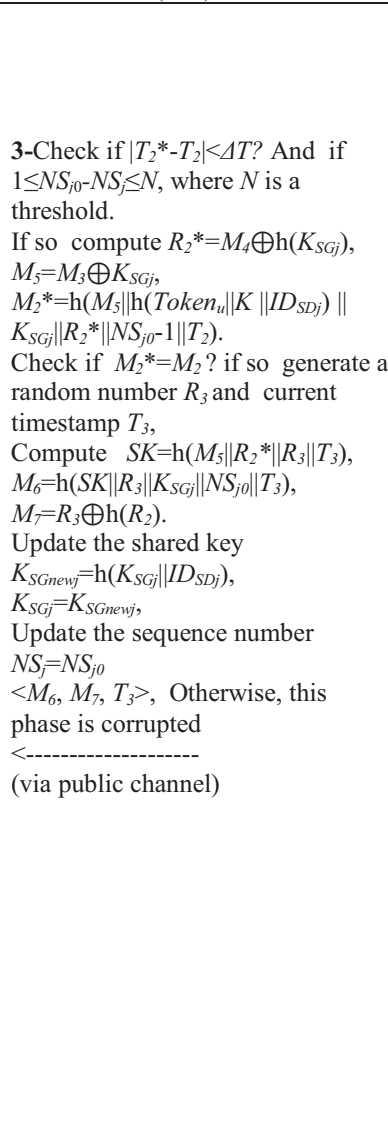 \\
\hline
\end{tabular}

D. Login and authentication phase

Once the registration process is completed, a user $U_{i}$ is now ready to login in the system. This phase achieves the goal of authentication among the $\mathrm{U}_{\mathrm{i}}, \mathrm{GW}$, and $\mathrm{SD}_{\mathrm{j}}$. Besides, at the end of the execution of this phase, a session key is established between $\mathrm{U}_{\mathrm{i}}$ and $\mathrm{SD}_{\mathrm{j}}$. This phase is explored in Table IV.

\section{SECURITY ANALYSIS}

Our scheme ensures many security properties and resists most popular attack. At first, TBLUA ensures the anonymity, thus any adversary $\mathrm{A}$ is unable to break the anonymity using the public messages. This is because the identities $I D_{i}$ and $I D_{S D j}$ are protected by $\mathrm{h}(\cdot)$. In addition, A needs to know $I D_{i}$, $I D_{S D j}$, the long secret key and Token $_{u}$ to compute $C I D_{i}, C I D_{S D j}$. Thus, our protocol can resist user impersonation attack. Furthermore, as A cannot retrieve $I D_{i}, I D_{S D j}$ and the shared Key $K_{S G}$ of $S D_{j}$, he/she cannot masquerade as a valid smart device and TBLUA resists the node impersonation attack. Besides, TBLUA is mainly designed to ensure the PFS service, let suppose A has obtained $K_{\mathrm{UG}}$ and $K_{S G}, \mathrm{He} / \mathrm{she}$ cannot get the session key $S K$. This is due to that after each successful session, $K_{U G}$ and $K_{S G}$ will be updated by one-way hash function. More than that, as we propose to use distinct shared secret keys $K_{S G j}, S D_{i}$ establishes a distinct session key with $U_{i}$, thus, although if A can capture a $S D_{i}$, all noncompromised devices still can communicate with the legitimate user $U_{i}$ with higher secrecy. Thus TBLUA withstands $S D_{j}$ node capture attacks. Moreover, our scheme can resist against token impersonation attack because without prior knowledge of $\mathrm{K}$, an adversary A cannot create a Token. And as the Token $_{u}$ is protected with a symmetric cipher function using $\mathrm{K}$, A cannot modify a valid token. Hence, TBLUA resists token modification attack.

\section{PERFORMANCE ANALYSIS}

In this section, we compare the communication and computation costs of the proposed scheme with three prior related works [5][8][12]. Since the distribution token phase is not used frequently and the cost is negligible (i.e. $\mathrm{GW} ; 4 T_{h}=$ $2 \mathrm{~ms}$ and Smart device: $3 T_{h}=1.5 \mathrm{~ms}$ ), we only concentrate on comparing login and authentication phase.

\section{A. Functionality comparison}

The functionality features of the existing schemes and the proposed scheme are compared in Table V. TBLUA can resist against various kinds of known attacks and fulfill the desirable security features such as PFS.

TABLE V. FunCtionality FEATURES COMPARISON

\begin{tabular}{|l|c|c|c|c|c|}
\hline Properties & {$[8]$} & {$[12]$} & & {$[5]$} & TBLUA \\
\hline Mutual Authentication & + & + & & + & + \\
\hline Key agreement & + & + & & + & + \\
\hline Intractability & - & + & & + & + \\
\hline User anonymity & - & + & & + & + \\
\hline SD. anonymity & - & + & & + & + \\
\hline Offline PW guessing & - & + & & + & + \\
\hline User impersonation & - & + & & + & + \\
\hline GW impersonation & - & + & & + & + \\
\hline SD impersonation & - & + & & + & + \\
\hline Privileged-insider & - & + & & + & + \\
\hline PFS & + & - & & - & + \\
\hline Replay attack & - & + & & + & + \\
\hline Stolen verifier & - & + & & + & + \\
\hline De-synchronization & - & - & & - & + \\
\hline Node capture & - & + & & + & + \\
\hline Token impersonation & N/A & N/A & & N/A & + \\
\hline Token modification & N/A & N/A & & N/A & + \\
\hline Note: N/A: Not Applicable & & & & &
\end{tabular}




\section{B. Computation costs comparison}

For the computation comparison, let the notations $T_{h}=0.5 \mathrm{~ms}$ be the time for one hashing operation and $T_{E n c}=T_{D e c}=8.7 \mathrm{~ms}$ be respectively the time for one encryption/decryption using symmetric cryptography operation and $T_{E C C}=T_{F E}=63.075 \mathrm{~ms}$ represent respectively the time for one elliptic curve cryptography one fuzzy extraction operation [4][5], we omit XOR operation due to its negligible computational cost. In Table VI, we provide computation cost separately for user, GW node and $\mathrm{S}$ of the login and authentication phase.

TABLE VI. COMPUTATION COSTS COMPARISON

\begin{tabular}{|c|c|c|c|}
\hline Scheme & User & GW & Smart Device \\
\hline$[\mathbf{8}]$ & $2 T_{E C C}+7 T_{h}=$ & $9 T_{h}$ & $2 T_{E C C}+5 T_{h}=$ \\
& $129,6 \mathrm{~ms}$ & $=4,5 \mathrm{~ms}$ & $128,6 \mathrm{~ms}$ \\
\hline$[\mathbf{1 2}]$ & $7 T_{h}+T_{D e c}+T_{E n c}=$ & $11 T_{h}+2 * T_{D e c}+2$ & $4 T_{h}+T_{D e c}+T_{E n c}=$ \\
& $20 \mathrm{~ms}$ & $* T_{E n c}=40.3 \mathrm{~ms}$ & $19.4 \mathrm{~ms}$ \\
\hline$[\mathbf{5}]$ & $T_{F E}+13 T_{h}+T_{D e c}+$ & $5 T_{h}+2 T_{D e c}+2 T_{E n}$ & $4 T_{h}+T_{D e c}+T_{E n c}=$ \\
& $T_{E n c}=87.0 \mathrm{~ms}$ & $c=37.3 \mathrm{~ms}$ & $19.4 \mathrm{~ms}$ \\
\hline TBLUA & $16 T_{h}=8 \mathrm{~ms}$ & $19 T_{h}+T_{D e c}=$ & $7 T_{\mathrm{h}}=3.5 \mathrm{~ms}$ \\
& & $18.2 \mathrm{~ms}$ & \\
\hline
\end{tabular}

\section{Communication costs comparison}

The communication costs of different existing schemes along with our proposed protocol are given in Table VII. It presents the comparison only for the phases that are executed frequently which are login and authentication phases. We assume that $I D_{i}$ is of length 160 bits, the identity of smart device node is 32 bits, random nonce is of 128 bits, symmetric encryption/decryption block size is of 128 bits (i.e., if we apply AES-128 algorithm [11]), timestamp is of 32 bits, sequence number is of 64 bits, and hash digest is of 160 bits (i.e. if SHA-1 hashing algorithm is applied [14]). For elliptic curve cryptography (ECC) based schemes, we consider a security level of 160-bit.

TABLE VII. COMMUNICATION COSTS COMPARISON

\begin{tabular}{|c|c|c|c|c|}
\hline Scheme & User & GW & Smart device & Total Cost \\
\hline [8] & 84 Byte & 64 Byte & 136 Byte & 284 Byte \\
\hline [12] & 80 Byte & 120 Byte & 40 Byte & 240 Byte \\
\hline [5] & 92 Byte & 168 Byte & 64 Byte & 324 Byte \\
\hline TBLUA & 84 Byte & 156 Byte & 44 Byte & 284 Byte \\
\hline
\end{tabular}

Fig.2 shows that the simulation results confirm the efficiency of our proposed scheme. In fact, our scheme has the lowest computation cost compared to benchmarking schemes and achieves a desirable communication overhead.
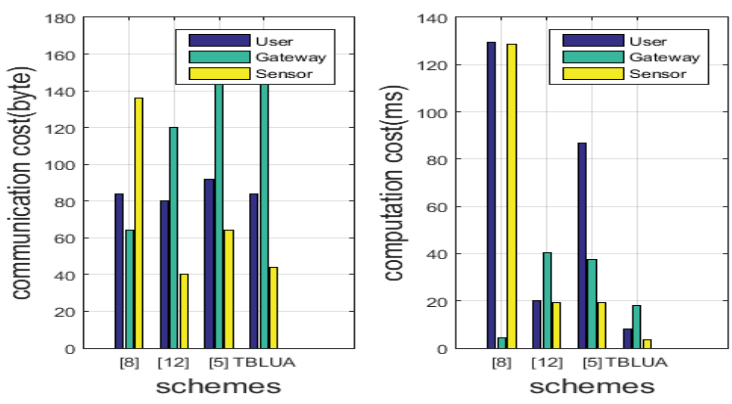

Fig. 2. Performance comparison

\section{CONCLUSION}

In this paper, we have proposed a lightweight authentication protocol based on token technique which provides an authentication for a period of time and response to the needs of modern cities. In fact, the proposed protocol
TBLUA is adopted in system reservation to ensure a mutual authentication between the communicating parties (User, GW, IoT device). Then, we demonstrated the trade-off between effectiveness and efficiency of the proposed scheme. From security perspective, it provides relatively more security features and high security level such as anonymity, Perfect Forward Secrecy, and resilience against the wellknown attacks. Furthermore, performance analysis proved that TBLUA has a low computation and communication overhead compared to benchmarking schemes. In future works, further results will be conducted along with a formal verification using the AVISPA tool.

\section{ACKNOWLEDMGELNT}

This work is achieved as part of the European project ITEA PARFAIT [15], which is partially funded by FEDER (European Regional Development Fund), BPIFRANCE, and the $\mathrm{BFC}$ region (Bourgogne-Franche-Comté).

\section{REFERENCES}

[1] K. T. Nguyen, M. Laurent, N. Oualha, "Survey on secure communication protocols for the Internet of Things", Ad Hoc Netw., vol. 32 , pp. 17-31, Sep. 2015

[2] M. A. Ferrag, L. A. Maglaras, H. Janicke, J. Jiang, and L. Shu, "Authentication Protocols for Internet of Things: A Comprehensive Survey," Security and Communication Networks, vol. 2017.

[3] O. O. Bamasag and K. Youcef-Toumi. "Towards Continuous Authentication in Internet of Things Based on Secret Sharing Scheme", In Proceedings of the WESS'15: Workshop on Embedded Systems Security (WESS'15). ACM, New York, NY, USA.

[4] S. Challa, M. Wazid, A. K. Das, N. Kumar, A. G. Reddy, E. J. Yoon, and K. Y. Yoo, "Secure Signature-Based Authenticated Key Establishment Scheme for Future IoT Applications," IEEE Access, vol. 5, pp. 3028-3043, 2017.

[5] M. Wazid, A. K. Das, V. Odelu, N. Kumar, M. Conti and M. Jo, "Design of Secure User Authenticated Key Management Protocol for Generic IoT Networks," in IEEE Internet of Things Journal, vol. 5, no. 1, pp. 269-282, Feb. 2018

[6] L. Xiong, D.Peng, T.Peng, H.Liang, and Z.Liu, -A lightweight anonymous authentication protocol with perfect forward secrecy for wireless sensor networks॥, Sensors, vol.17, no.11, pp.2681:1- 28,2017.

[7] M. Turkanovic, B. Brumen, and M. Holbl, "A novel user authentication and key agreement scheme for heterogeneous ad hoc wireless sensor networks, based on the Internet of Things notion," Ad Hoc Networks, vol. 20, pp. $96-112,2014$

[8] C. C. Chang and H. D. Le, "A Provably Secure, Efficient and Flexible Authentication Scheme for Ad hoc Wireless Sensor Networks," IEEE Transactions on Wireless Communications, vol. 15, no. 1, pp. 357-366, 2016.

[9] P. Gope and T. Hwang, "A Realistic Lightweight Anonymous Authentication Protocol for Securing Real-Time Application Data Access in Wireless Sensor Networks," in IEEE Transactions on Industrial Electronics, vol. 63, no. 11, pp. 7124-7132, Nov. 2016.

[10] N. Khalil, M. R. Abid, D. Benhaddou, and M. Gerndt, "Wireless sensors networks for Internet of Things," in IEEE Ninth International Conference on Intelligent Sensors, Sensor Networks and Information Processing (ISSNIP), Singapore, 2014, pp. 1-6.

[11] “Advanced Encryption Standard (AES)," FIPS PUB 197, National Institute of Standards and Technology (NIST), U.S. Department of Commerce, November 2001. http://csrc.nist.gov/publications/fips/fips197/fips-197.pdf. Accessed on April 2018.

[12] Y. Lu, L. Li, H. Peng, et al. "An Energy Efficient Mutual Authentication and Key Agreement Scheme Preserving Anonymity for Wireless Sensor Networks," Sensors, vol. 16, no. 6, article no. 837, 2016.

[13] T. S. Messerges, E. A. Dabbish, and R. H. Sloan, "Examining smartcard security under the threat of power analysis attacks," IEEE Transactions on Computers, vol. 51, no. 5, pp. 541-552, 2002.

[14] "Secure Hash Standard," FIPS PUB 180-1, National Institute of Standards and Technology (NIST), U.S. Department of Commerce, April 1995.

[15] http://www.itea3-parfait.com/. 